\title{
PERFORMANCE ANALYSIS OF THE CEPSTRAL TECHNIQUE FOR FREQUENCY-DOMAIN OPTICAL-COHERENCE TOMOGRAPHY
}

\author{
S. Chandra Sekhar* and Michael Unser
}

Biomedical Imaging Group

Ecole polytechnique fédérale de Lausanne (EPFL), Switzerland

Emails: \{chandrasekhar.seelamantula, michael.unser\} @epfl.ch

\begin{abstract}
Recently, we proposed a noniterative cepstral technique for exact signal recovery in frequency-domain optical-coherence tomography. In this paper, we address the influence of measurement noise on the performance of the method. We derive analytical expressions for the bias and variance of the tomogram under a small noise approximation, and show that our technique yields unbiased and consistent estimators, which have a variance that is proportional to that of the noise and inversely proportional to the data size. We present simulation results to confirm the theoretical derivations. We also derive approximate Cramér-Rao bounds (CRBs) on the achievable accuracy of reconstruction.
\end{abstract}

Index Terms - frequency-domain optical-coherence tomography (FDOCT), cepstrum, bias, variance, mean square error, Cramér-Rao bound.

\section{INTRODUCTION}

Frequency-domain optical-coherence tomography (FDOCT) is now widely recognized as a fast technique for non-invasive three-dimensional imaging of biological specimens. With a penetration depth of 2-3 $\mathrm{mm}$ in tissue, resolution of the order of a few micrometers, higher sensitivity [1] and faster imaging speed [2], it has superseded its time-domain counterpart. The quality of tomograms that one achieves is closer to that obtained by high-resolution and invasive methodologies such as histology. FDOCT has been successfully applied in tissueimaging, dermatology, and opthalmology [2, 3, 4, 5], with some of them being in vivo.

In FDOCT, the depth information is acquired within a single exposure by means of spectrometer detection. A standard approach to reconstruct the tomogram is to apply the inverse Fourier transform [6]. However, it is known to introduce autocorrelation artifacts and complex-conjugate ambiguity. The latter can be suppressed by placing the zero-delay plane of the interferometer outside the specimen. In our earlier work

* This work is supported by the Center for Biomedical Imaging (CIBM) of the Geneva-Lausanne universities and the EPFL, the Hasler, Leenards and Louis-Jeantet foundations.

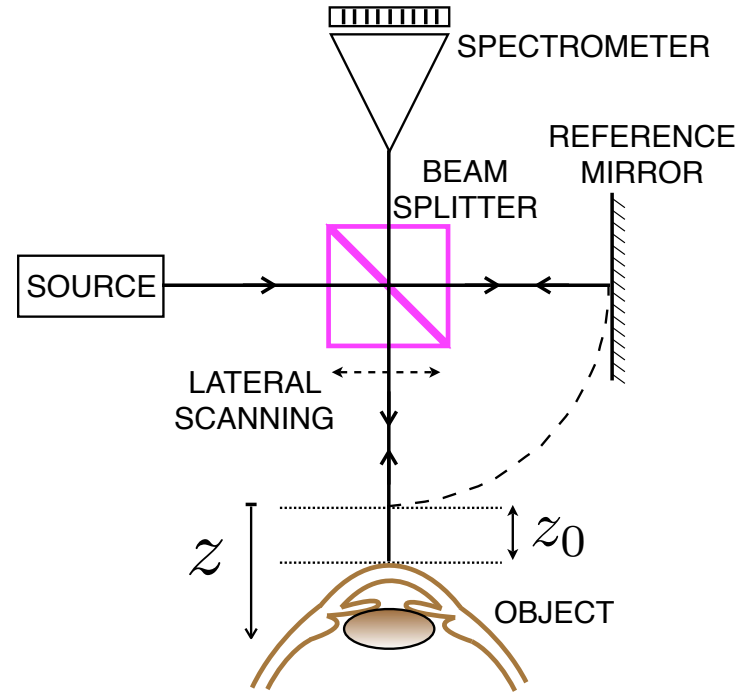

Fig. 1. Schematic of the Fourier-domain optical-coherence tomography system.

[7], we proposed a new algorithm to suppress the remaining autocorrelation artifacts. In this paper, we analyze its noise sensitivity, derive expressions for the bias and variance and also corroborate the analytical predictions with Monte-Carlo analysis.

\section{SIGNAL ACQUISITION AND ARTIFACT-FREE TOMOGRAM RECONSTRUCTION}

Figure 1 displays a typical FDOCT experimental setup. It is a Michelson interferometric configuration comprising an object arm and a reference arm. The light source is chosen to possess a broad spectrum so as to achieve high axial resolution. The source output is split into two beams, one of which is directed towards the object arm and the other towards the reference arm. A broadband mirror placed in the reference arm acts as a perfect reflector. The backscattered light from the specimen placed in the object arm is coherently amplified by 
the reference-arm signal, when the two are suitably combined. The resulting interference signal is recorded by a spectrometer, as a function of the wavelength $\lambda$. These measurements are then mapped onto the wavenumber scale $k=\frac{2 \pi}{\lambda}$. The measured signal then takes the form

$$
I(\omega)=S(\omega)|1+A(\omega)|^{2}
$$

where $A(\omega)=\int_{0}^{\infty} a(z) e^{-\mathrm{j} \omega z} \mathrm{~d} z, \omega=-2 k n, n$ being the refractive index of the specimen, and where $a(z)$ is the scattering function. The objective is to recover $a(z)$ from $I(\omega)$. In [7], we proposed a new technique to accomplish this task. It is summarized as follows: Given the measurements $I(\omega)$ and $S(\omega)$, we compute the cepstrum $c(z)=\mathcal{F}^{-1}\{\log (I / S)\}(z)$, where $\mathcal{F}$ denotes the Fourier transform operator. The causal part of $c(z)$ is retained by multiplying with a unit-step function $u(z)$ to yield $c^{+}(z)$. Next, we compute the Fourier transform of $c^{+}(z)$ to obtain $C^{+}(\omega)$. The nonlinear transformation $\exp \left(C^{+}(\omega)\right)-1$ yields $A(\omega)$ from which $a(z)$ is obtained by an inverse Fourier transform as $a(z)=\mathcal{F}^{-1}\{A\}(z)$.

In the following analysis, we consider a discrete setting in which $a_{k}, A_{k}, S_{k}$ and $I_{k}$ denote the samples of $a(z), A(\omega), S(\omega)$ and $I(\omega)$ respectively.

\section{BIAS AND VARIANCE OF THE CEPSTRAL RECONSTRUCTION TECHNIQUE}

Our model for the noisy signal is that of a deterministic signal in random additive noise $W_{k}$, and is given by

$$
I_{k}=S_{k}\left|1+A_{k}\right|^{2}+W_{k}, 0 \leq k \leq N-1,
$$

where $\left|A_{k}\right| \ll 1$, and where $W_{k}$ is assumed to have a Gaussian distribution with zero mean and variance $\sigma_{w}^{2}$. Rewriting (2) as

$$
I_{k}=S_{k}\left|1+A_{k}\right|^{2}\left(1+\frac{W_{k}}{S_{k}\left|1+A_{k}\right|^{2}}\right), 0 \leq k \leq N-1,
$$

and taking its logarithm, we get

$\log I_{k}=\log S_{k}+\log \left|1+A_{k}\right|^{2}+\log \left(1+\frac{W_{k}}{S_{k}\left|1+A_{k}\right|^{2}}\right)$.

Under the assumption that the noise is small in comparison to the signal, we invoke the approximation $\log (1+x)=x+$ $\mathcal{O}\left(x^{2}\right)$, for $|x|<1$, where $\mathcal{O}$ denotes the Landau symbol. Using the approximation, we rewrite (4) as

$$
\log I_{k}-\log S_{k}=\log \left|1+A_{k}\right|^{2}+\alpha_{k} W_{k},
$$

where $\alpha_{k}=\frac{1}{S_{k}\left|1+A_{k}\right|^{2}}$, and where the higher-order terms are not shown for the sake of brevity. Taking the inverse discrete Fourier transform (IDFT) on both sides of (5), we obtain the cepstrum

$$
\begin{aligned}
\widehat{c}_{m} & =\frac{1}{N} \sum_{k=0}^{N-1}\left(\log \left|1+A_{k}\right|^{2}+\alpha_{k} W_{k}\right) e^{\mathrm{j} \frac{2 \pi}{N} k m}, \\
& =c_{m}+\frac{1}{N} \sum_{k=0}^{N} \alpha_{k} W_{k} e^{\mathrm{j} \frac{2 \pi}{N} k m},
\end{aligned}
$$

where $c_{m}$ is the cepstrum in the absence of noise. The causal cepstrum is given by

$$
\widehat{c}_{m}^{+}=\left\{\begin{aligned}
\widehat{c}_{m} & \text { if } 0 \leq m \leq \frac{N}{2}-1 \\
0 & \text { if } \frac{N}{2} \leq m \leq N-1 .
\end{aligned}\right.
$$

The DFT of $\left\{\widehat{c}_{m}^{+}, 0 \leq m \leq N-1\right\}$ is

$$
\begin{aligned}
\widehat{C}_{k}^{+} & =\sum_{m=0}^{N / 2-1} \widehat{c}_{m} e^{-\mathrm{j} \frac{2 \pi}{N} k m}, 0 \leq k \leq N-1 \\
& =C_{k}^{+}+\frac{1}{N} \sum_{m=0}^{N / 2-1} \sum_{\ell=0}^{N-1} \alpha_{\ell} W_{\ell} e^{\mathrm{j} \frac{2 \pi}{N} \ell m} e^{-\mathrm{j} \frac{2 \pi}{N} k m}, \\
& =C_{k}^{+}+\frac{1}{N} \sum_{\ell=0}^{N-1} \alpha_{\ell} W_{\ell} \underbrace{\sum_{m=0}^{N / 2-1} e^{\mathrm{j} \frac{2 \pi}{N}(\ell-k) m}}_{\beta_{\ell, k}} \\
& =C_{k}^{+}+\frac{1}{N} \sum_{\ell=0}^{N-1} \alpha_{\ell} \beta_{\ell, k} W_{\ell}
\end{aligned}
$$

where the $\beta_{\ell, k}$ are deterministic. When the $\widehat{C}_{k}^{+}$are raised to the exponent of the Naperian base $e$, we get,

$$
\begin{aligned}
\widehat{A}_{k} & =\exp \left(\widehat{C}_{k}^{+}\right)-1 \\
& =\exp \left(C_{k}^{+}+\frac{1}{N} \sum_{\ell=0}^{N-1} \alpha_{\ell} \beta_{\ell, k} W_{\ell}\right)-1, \\
& \approx \exp \left(C_{k}^{+}\right)\left(1+\frac{1}{N} \sum_{\ell=0}^{N-1} \alpha_{\ell} \beta_{\ell, k} W_{\ell}\right)-1,
\end{aligned}
$$

where the approximation $e^{x}=1+x+\mathcal{O}\left\{x^{2}\right\},|x|<1$, has been used. This approximation is valid under the small-noise assumption. Hence,

$$
\widehat{A}_{k}=A_{k}+\exp \left(C_{k}^{+}\right)\left(\frac{1}{N} \sum_{\ell=0}^{N-1} \alpha_{\ell} \beta_{\ell, k} W_{\ell}\right) .
$$

The scattering function estimated by applying the IDFT to the $\left\{\widehat{A}_{k}, 0 \leq k \leq N-1\right\}$ is given as

$$
\begin{aligned}
\widehat{a}_{m} & =a_{k}+\frac{1}{N^{2}} \sum_{\ell=0}^{N-1} \alpha_{\ell} W_{\ell} \underbrace{\sum_{k=0}^{N-1} \exp \left(C_{k}^{+}\right) \beta_{\ell, k} e^{\mathrm{j} \frac{2 \pi}{N} k m}}_{\gamma_{\ell, m}}, \\
& =a_{m}+\frac{1}{N^{2}} \sum_{\ell=0}^{N-1} \alpha_{\ell} \gamma_{\ell, m} W_{\ell}, 0 \leq m \leq \frac{N}{2}-1,(11)
\end{aligned}
$$


where the $\gamma_{\ell, m}$ are deterministic. Applying the expectation operator $\mathcal{E}$ on both sides of (11), we get

$$
\mathcal{E}\left\{\widehat{a}_{m}\right\}=a_{m},
$$

where we have used the assumption that the noise has zero mean. Thus, our technique yields unbiased estimates. The variance of $\widehat{a}_{m}$ is

$$
\mathcal{E}\left\{\left|\widehat{a}_{m}-\mathcal{E}\left\{\widehat{a}_{m}\right\}\right|^{2}\right\}=\frac{\sigma_{w}^{2}}{N^{4}} \sum_{\ell=0}^{N-1} \alpha_{\ell}^{2}\left|\gamma_{\ell, m}\right|^{2}
$$

\section{BIAS AND VARIANCE OF THE FOURIER RECONSTRUCTION TECHNIQUE}

In the conventional Fourier reconstruction technique, it is a common practice to subtract the measured source spectrum $S_{k}$ from the measurements $I_{k}$, before applying the IDFT. This operation boosts the information-carrying interference fringes Thus, we have

$$
\begin{aligned}
H_{k} & =S_{k}\left|1+A_{k}\right|^{2}+W_{k}-S_{k}, 0 \leq k \leq N-1, \\
& =2 S_{k} \operatorname{Re}\left\{A_{k}\right\}+S_{k}\left|A_{k}\right|^{2}+W_{k} .
\end{aligned}
$$

The IDFT of $\left\{H_{k}, 0 \leq k \leq N-1\right\}$ is given by

$h_{m}=\frac{1}{N} \sum_{k=0}^{N-1}\left(2 S_{k} \operatorname{Re}\left\{A_{k}\right\}+S_{k}\left|A_{k}\right|^{2}+W_{k}\right) e^{\mathrm{j} \frac{2 \pi}{N} k m}$.

Considering only the causal part of $h_{m}$, we get $\widehat{a}_{m}=(s *$ $a)_{m}+\left(s * r_{a a}\right)_{m}+w_{m}$, where $s_{m}$ is the source autocorrelation, $w_{m}$ is the noise, and $r_{a a}$ is the autocorrelation of the scattering function, all of these being in the spatial domain. The source has a convolutional effect and the noise is additive. The expectation of $\widehat{a}_{m}$ is given by

$$
\mathcal{E}\left\{\widehat{a}_{m}\right\}=(s * a)_{m}+\left(s * r_{a a}\right)_{m},
$$

which implies that the estimates are biased. The variance is given by $\mathcal{E}\left\{\left|\widehat{a}_{m}-\mathcal{E}\left\{\widehat{a}_{m}\right\}\right|^{2}\right\}=\frac{\sigma_{w}^{2}}{N}$.

\section{CRAMÉR-RAO BOUNDS}

We derive the CRBs corresponding to $A_{k}=\sum_{m=0}^{N-1} a_{m} e^{-\mathrm{j} \frac{2 \pi}{N} k m}$ in (2), where the $a_{m}$ are assumed to be real. The entries of the Fisher information matrix $\mathbf{J}$ are given by

$$
\mathbf{J}_{\ell, \ell^{\prime}}=\mathcal{E}\left\{\frac{\partial \log f}{\partial a_{\ell}} \cdot \frac{\partial \log f}{\partial a_{\ell^{\prime}}}\right\}, 0 \leq \ell, \ell^{\prime} \leq N-1,
$$

where $f$ is the probability density function of $\left\{I_{k}, 0 \leq k \leq\right.$ $N-1\}$. After some computations, we arrive at

$$
\mathbf{J}_{\ell, \ell^{\prime}}=\frac{1}{4 \sigma_{w}^{2}} \sum_{k=0}^{N-1} \eta_{\ell, k} \eta_{\ell^{\prime}, k}
$$

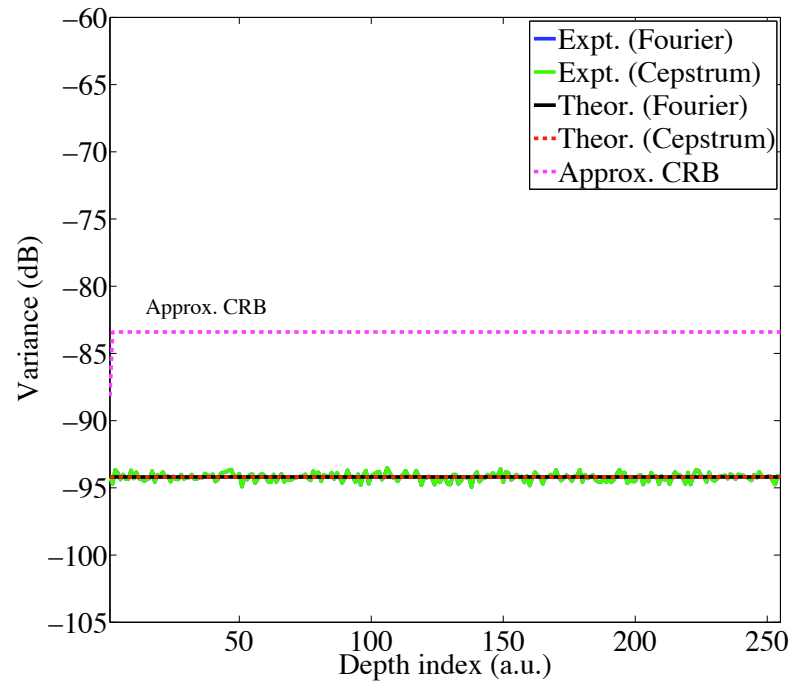

Fig. 2. A comparison of approximate theoretical variances vs. those obtained by simulation $(\mathrm{SNR}=20 \mathrm{~dB})$. The variances of the cepstral and the Fourier techniques are nearly identical.

where

$$
\begin{aligned}
\eta_{\ell, k}= & -2 S_{k} \cos \left(\frac{2 \pi k \ell}{N}\right) \\
& -2 S_{k} \sum_{p=0}^{N-1} a_{p} \cos \left(\frac{2 \pi(\ell-p) k}{N}\right) .
\end{aligned}
$$

$\left(\mathbf{J}^{-1}\right)_{k, k}$ gives a bound on the variance of $\widehat{a}_{k}$, however, it is not easy to compute it in a closed form. Numerical calculations show that $\mathbf{J}$ becomes singular even for small values of $N$. We propose to use the pseudoinverse of $\mathbf{J}$ instead, keeping in mind that this may yield an inaccurate bound.

\section{MONTE-CARLO ANALYSIS}

Deconvolution of the source spectrum is implicit in our technique, which is not the case with the Fourier technique. Therefore, to make a fair comparison, we set the source spectrum to unity within the measurement bandwidth. We synthesize a one-dimensional scattering function corresponding to a threelayered specimen. Next, we generate 500 realizations of white Gaussian noise and compute estimates of the scattering function by using the cepstral as well as the standard Fourier techniques. We repeat the experiment for different values of signalto-noise ratio (SNR), and corresponding to each value, we compute the variance of the estimator. Figures 2 and 3 show the variances of the estimates given by the two techniques corresponding to $\mathrm{SNR}=20 \mathrm{~dB}$ and $40 \mathrm{~dB}$, respectively. The variances at an arbitrarily-chosen depth are shown in Fig. 4, 


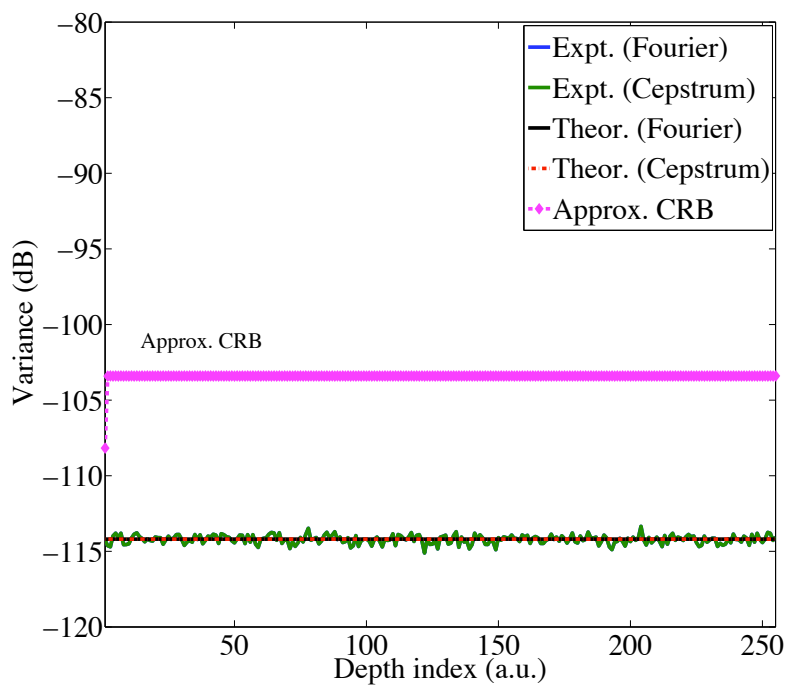

Fig. 3. A comparison of approximate theoretical variances vs. those obtained by simulation $(\mathrm{SNR}=40 \mathrm{~dB})$. The variances of the cepstral and the Fourier techniques are nearly identical.

together with the theoretical variances and the CRBs. Note that the two techniques have almost identical variances. The pseudoinverse-based CRB is quite pessimistic, because it underestimates the performance of the best estimator. The two techniques are significantly better than the bound.

\section{CONCLUSION}

We addressed the noise performance of a new cepstral reconstruction algorithm for frequency-domain optical-coherence tomography [7]. We also compared the noise-sensitivity with respect to the conventional Fourier technique. Inspite of it being nonlinear, our technique has a variance that is as low as that of the linear technique. This implies a high consistency of reconstruction across different realizations of the scattering function, which is a useful feature in practice. The added advantage is that our technique suppresses the autocorrelation artifacts and yields unbiased estimates. Comparisons to iterative reconstruction techniques will be reported separately.

\section{REFERENCES}

[1] R. A. Leitgeb, C. K. Hitzenberger, and A. F. Fercher, "Performance of Fourier-domain vs. timedomain optical-coherence tomography," Opt. Express, vol. 11, no. 8, pp. 889-894, April 2003.

[2] A. F. Fercher, C. K. Hitzenberger, G. Kamp, and S. Y. El-Zaiat, "Measurement of intraocular distances by

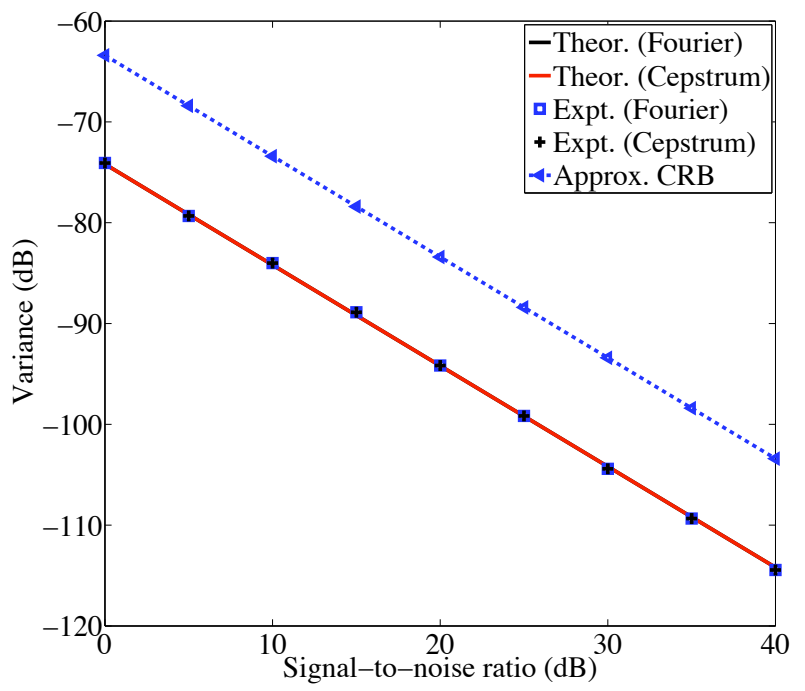

Fig. 4. Variances of the two estimators, at an arbitrarily chosen depth, as a function of the SNR. The variances of the cepstral and the Fourier techniques are nearly identical.

backscattering spectral interferometry," Opt. Comm., vol. 117, pp. 43-48, 1995.

[3] N. A. Nassif, B. Cense, B. H. Park, M. C. Pierce, S. H. Yun, B. E. Bouma, G. J. Tearney, T. C. Chen, and J. F. de Boer, "In vivo high-resolution video-rate spectral-domain optical-coherence tomography of the human retina and optic nerve," Opt. Express, vol. 12, no. 3, pp. 367-376, 2004.

[4] M. Wojtkowski, T. Bajraszewski, I. Gorczynska, P. Targowski, A. Kowalczyk, W. Wasilewski, and C. Radzewicz, "Ophthalmic imaging by spectral opticalcoherence tomography," Am. J. of Ophthalmology, vol. 138, no. 3, pp. 412-419, 2004.

[5] U. Schmidt-Erfurth, R. A. Leitgeb, S. Michels, B. Povazay, S. Sacu, B. Hermann, C. Ahlers, H. Sattmann, C. Scholda, A. F. Fercher, and W. Drexler, "Threedimensional ultrahigh-resolution optical-coherence tomography of macular diseases," Invest Ophthalmol. Vis. Sci., vol. 46, no. 9, pp. 3393-3402, 2005.

[6] B. E. Bouma and G. J. Tearney, Eds., Handbook of Optical Coherence Tomography, Marcel Dekker, Inc., 2002.

[7] S. C. Sekhar, R. A. Leitgeb, M. L. Villiger, A. H. Bachmann, T. Blu, and M. Unser, "Noniterative exact signal recovery in frequency-domain optical-coherence tomography," in Proc. of the Fourth IEEE Intl. Symp. on Biomed. Imaging: From Nano to Macro (ISBI'07), Arlington VA, USA, April 12-15, 2007, pp. 808-811. 